MZ-TH/98-12

hep-ph/9805224

March 1998

\title{
A new technique for computing the spectral density of sunset-type diagrams: integral transformation in configuration space
}

\author{
S. Groote, ${ }^{1}$ J.G. Körner ${ }^{1}$ and A.A. Pivovarov ${ }^{1,2}$ \\ ${ }^{1}$ Institut für Physik, Johannes-Gutenberg-Universität, \\ Staudinger Weg 7, D-55099 Mainz, Germany \\ ${ }^{2}$ Institute for Nuclear Research of the \\ Russian Academy of Sciences, Moscow 117312
}

\begin{abstract}
We present a new method to investigate a class of diagrams which generalizes the sunset topology to any number of massive internal lines. Our attention is focused on the computation of the spectral density of these diagrams which is related to manybody phase space in $D$ dimensional space-time. The spectral density is determined by the inverse $K$-transform of the product of propagators in configuration space. The inverse $K$-transform reduces to the inverse Laplace transform in any odd number of space-time dimensions for which we present an explicit analytical result.
\end{abstract}




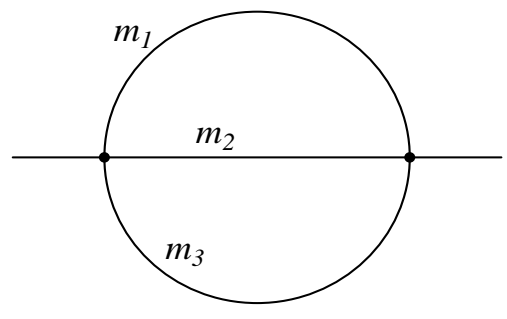

(a)

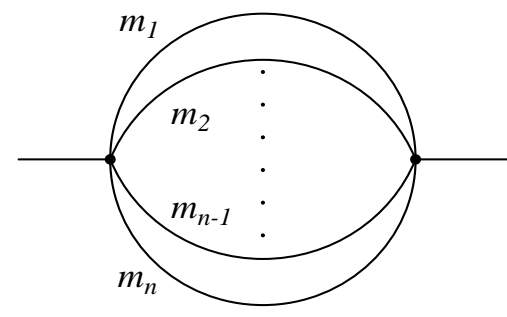

(b)

Figure 1: (a) a sunset diagram with three different masses $m_{1}, m_{2}$ and $m_{3}$ (b) general topology of the class of water melon diagrams

Recently there has been renewed interest in the calculation of sunset-type diagrams with massive internal lines (as some of the latest refs. see for example [1, 2, 3, [4]). The simplicity of the sunset-type topology has a special appeal since sunset-type diagrams can be regarded as a laboratory for testing new methods in the computation of diagrams with massive particles (see e.g. [5]). For phenomenological applications one needs to avail of efficient numerical algorithms to compute the sunset-type diagrams (see e.g. [6]). In [7] we have derived a one-dimensional integral representation for a class of diagrams that generalizes the sunset topology to any number of internal lines (we call them water melon diagrams). We believe that the method described in [7] and in the present paper completely solves the problem of calculating the class of sunset-type diagrams.

In this note we present a new representation for the spectral density of water melon diagrams through an integral transformation in configuration space. It is given by an inverse $K$-transform of the product of propagators of internal lines that constitutes the water melon diagram. The representation simplifies to the inverse of the standard Laplace transform in any odd number of space-time dimensions for which we present a concise analytical result. The spectral density of water melon diagrams is related to the manybody phase space integral in $D$ dimensional space-time for which we find novel results.

The sunset diagram proper represents the leading order perturbation theory correction 
to the propagator in $\phi^{4}$-theory, i.e. it is a two-loop insertion with three internal lines (see Fig. 1(a)). A straightforward generalization of this topology (water melon diagram) is a correction to the propagator in the $\phi^{n+2}$-theory that contains $n$ loops and $n+1$ internal lines (see Fig. 1(b)). As an example take the leading quantum corrections of higher order chiral perturbation theory for pseudoscalar mesons where such diagrams occur. Thus we describe as a generic case of the water melon diagram the correlator of two field monomials $j_{n}(x)=: \phi_{1, \mu_{1}} \ldots \phi_{n, \mu_{n}}$ : taken in the normal ordering form where $\phi_{1, \mu}$ is a derivative of the field $\phi_{1}$ with a multi index $\mu=\left\{\mu_{1}, \ldots, \mu_{p}\right\}$. We leave aside trivial generalizations that would result from the inclusion of derivatives and the emission/absorption of additional external particles with vanishing momentum. These would merely result in the doubling of the propagators to which they are attached and thus are easily included in our general approach. Returning to the scalar case the general representation of the scalar polarization function corresponding to water melon diagrams is given by

$$
\Pi(x)=\prod_{i=1}^{n} D\left(x, m_{i}\right)
$$

where $D(x, m)$ is the configuration space propagator of a particle with mass $m$ in $D$ dimensional (Euclidean) space-time [8]

$$
D(x, m)=\frac{1}{(2 \pi)^{D}} \int \frac{e^{i p_{\mu} x^{\mu}} d^{D} p}{p_{\mu} p^{\mu}+m^{2}}=\frac{(m x)^{\lambda} K_{\lambda}(m x)}{(2 \pi)^{\lambda+1} x^{2 \lambda}}
$$

where $D=2 \lambda+2$, and $K_{\lambda}(z)$ is a McDonald function (a modified Bessel function of the third kind, see e.g. [9]). When we refer to a specific space-time dimension $D$ later on we add the space-time index to the propagator function, i.e. we write $D_{D}(x, m)$ for the propagator function. The propagator depends only on the length $x$ of the $D$-dimensional vector $\left(x^{\mu}\right), x=\sqrt{x_{\mu} x^{\mu}}$ (a times we shall also use the notation $x$ for the vector itself).

The representation Eq. (11) contains the complete information about the class of water melon diagrams and is the starting point for all further investigations.

For some applications one needs the Fourier transform of the polarization function 
which is defined by

$$
\tilde{\Pi}(p)=\int \Pi(x) e^{i p_{\mu} x^{\mu}} d^{D} x=\int\left\langle T j_{n}(x) j_{m}(0)\right\rangle e^{i p_{\mu} x^{\mu}} d^{D} x
$$

The analytic structure of the Fourier transform $\tilde{\Pi}(p)$ is determined by the Källen-Lehmann representation

$$
\tilde{\Pi}(p)=\int_{0}^{\infty} \frac{\rho(s) d s}{s+p^{2}}-(\text { subtractions })
$$

with the appropriate number of subtractions for the regularization of ultraviolet divergences, if necessary. The spectral density $\rho(s)$ or the discontinuity across the cut in the complex plane of the analytic function $\tilde{\Pi}(p)$ is of interest for various physics applications. We mention, though, that in the Euclidean region the function $\tilde{\Pi}(p)$ itself also has some applications. For these applications the values of $\tilde{\Pi}(p)$ can easily be found from the representation Eq. (4) or directly from Eq. (3) [7]. Here we shall focus our attention on the spectral decomposition of the water melon diagrams which in turn is connected with the particle content of the underlying theory.

The analytic structure of the correlator $\Pi(x)$ (or the spectral density of the corresponding polarization operator) can be determined directly in configuration space without having to compute its Fourier transform first. The dispersion representation (or the spectral decomposition) of the polarization function in configuration space has the form

$$
\Pi(x)=\int_{0}^{\infty} \rho(s) D(x, s) d s
$$

where $D(x, s)$ is the scalar propagator with the mass $m=\sqrt{s}$. This representation was used for sum rules applications in [10, 11] where the spectral density for the two-loop sunset diagram was found in two-dimensional space-time [12. With the explicit form of the propagator in configuration space Eq. (2) the representation in Eq. (5) turns into a particular example of the Hankel transform, namely the $K$-transform [13, 14. Up to inessential factors of $x$ and $\sqrt{s}$, Eq. (5) reduces to the generic form of the $K$-transform for 
a conjugate pair of functions $f$ and $g$,

$$
g(y)=\int_{0}^{\infty} f(x) K_{\nu}(x y) \sqrt{x y} d x
$$

The inverse of this transform is known to be given by

$$
f(x)=\frac{1}{\pi i} \int_{c-i \infty}^{c+i \infty} g(y) I_{\nu}(x y) \sqrt{x y} d y
$$

where $I_{\nu}(x)$ is a modified Bessel function of the first kind and the integration runs along a vertical contour in the complex plane to the right of the right-most singularity of the function $g(y)$. In order to obtain a representation for the spectral density $\rho(s)$ of water melon diagrams in general $D$-dimensional space-time one needs to apply the inverse $K$ transform to the particular case Eq. (51). One has

$$
m^{\lambda} \rho\left(m^{2}\right)=\frac{(2 \pi)^{\lambda}}{i} \int_{c-i \infty}^{c+i \infty} \Pi(x) x^{\lambda+1} I_{\lambda}(m x) d x
$$

The transform Eq. (8) solves the problem of determinating the spectral density of water melon diagrams by reducing it to the computation of a one-dimensional integral for a general water melon diagram with any number of internal lines and different masses. Below we discuss some technicalities concerning the efficient evaluation of the contour integral.

The analytic structure of the correlator in Eq. (11) is now explicit using the representation given by Eq. (8) and exhibits the distribution nature of the spectral density $\rho(s)$. For odd-dimensional space-time the representation in Eq. (5) reduces to the Laplace transformation. To obtain the spectral density (the function $f(x)$ in this particular example) one can use Eq. (17). For energies below threshold it is possible to close the contour of integration to the right. With the appropiate choice of the constant $c$ as specified above the closed contour integration gives zero due to the absence of singularities in the relevant domain of the right semi-plane. By closing the contour of integration to the left and keeping only that part of the function $I_{\nu}(z)$ which is exponentially falling for $\operatorname{Re}(z)<0$ one can obtain another convenient integral representation for the spectral density when the energy 
is above threshold. The only singularities within the closed contour are then poles at the origin (in odd-dimensional space-time) and the evaluation of the integral can be done by determining the corresponding residues. These are purely algebraic manipulations, the simplicity of which also explain the simplicity of the computations in odd-dimensional space time. For a small number of internal lines $n$ the spectral density can also be found by using the convolution formulae for the spectral densities of a smaller number of particles (see e.g. 15]). For large $n$ the computations described in 15 become quite cumbersome and the technique suggested in the present paper is much more convenient. For example, in odd-dimensional space-time our techniques give the result for water melon diagrams in terms of elementary functions. In three space-time dimensions our results can be used to compute the phase-space integrals for particles in jets where the momentum along the direction of the jet is fixed [16]. Another application can be found in three dimensional QCD which emerges as the high temperature limit of the ordinary theory of strong interactions for the quark-gluon plasma (see e.g. [17, 18, 19, 20]).

For even-dimensional space-time the analytic structure of $\Pi(x)$ in Eq. (1) is more complicated: there is a cut along the negative semi-axis which prevents a straightforward evaluation by simply closing the contour of integration to the left. The discontinuity along the cut is, however, well known and includes only Bessel functions that appear in the product of propagators for the polarization function. Therefore the representation (8) is essentially equivalent to the direct analytic continuation of the Fourier transform [7] but may be more convenient for numerical treatment because there is no oscillating integrand in (8).

Note also that the expression given by Eq. (1) can have non-integrable singularities at small $x$ for a sufficiently large number of propagators when $D>2$ [21]. Therefore the computation of its Fourier transform requires regularization or subtractions [7]. The spectral density itself is finite (the structure of the water melon diagrams is very simple and there are no subdivergences when one employs a properly defined $R$-operation [21]) 
and thus requires no regularization. The explicit representation for the spectral density within the more traditional approach reads $[7]$

$$
\begin{gathered}
\left.2 \pi i \operatorname{Disc} \tilde{\Pi}(p)\right|_{p^{2}=-s} \\
=\int_{0}^{\infty}\left(\frac{2 \pi \xi}{s}\right)^{\lambda+1} J_{\lambda}(\xi)\left[e^{-i \pi(\lambda+1)} \prod_{i=1}^{n} \frac{1}{4}\left(\frac{m_{i} \sqrt{s}}{2 \pi \xi}\right)^{\lambda} e^{i \pi(\lambda+1 / 2)} H_{\lambda}^{(1)}\left(\frac{m_{i} \xi}{\sqrt{s}}\right)\right. \\
\left.\quad-e^{i \pi(\lambda+1)} \prod_{i=1}^{n} \frac{1}{4}\left(\frac{m_{i} \sqrt{s}}{2 \pi \xi}\right)^{\lambda} e^{-i \pi(\lambda+1 / 2)} H_{\lambda}^{(2)}\left(\frac{m_{i} \xi}{\sqrt{s}}\right)\right] d \xi
\end{gathered}
$$

where one has taken the discontinuity of the Fourier transform across the physical cut for $p^{2}=-s \pm i 0, s>0$. The analytic continuation is done according to [ 8

$$
K_{\lambda}(z)=\frac{\pi i}{2} e^{\frac{\pi}{2} \lambda i} H_{\lambda}^{(1)}(i z)
$$

with $H_{\lambda}^{(1,2)}(z)$ being the Hankel functions, $H_{\lambda}^{(1)}(z)=\left(H_{\lambda}^{(2)}(z)\right)^{*}$ for real $z$ and $\lambda$. This is an alternative representation of the spectral density, and in some instances this representation can be more convenient for numerical treatment.

In the following we present some explicit examples of applying the technique of computing the spectral density of water melon diagrams on the basis of integral transformations in configuration space.

First a remark about the mass degenerate one-loop case is in order. All necessary integrals (both for the direct and the inverse $K$-transform) involve no more than the product of three Bessel functions which can be found in a standard collection of formulas for special functions (see e.g. [8]). The spectral density in $D$-dimensional space-time (for two internal lines with equal masses) can be computed to be

$$
\rho(s)=\frac{\left(s-4 m^{2}\right)^{\lambda-1 / 2}}{2^{4 \lambda+1} \pi^{\lambda+\frac{1}{2}} \Gamma\left(\lambda+\frac{1}{2}\right) \sqrt{s}}, \quad s>4 m^{2} .
$$

This formula is useful since it can be used to test the limiting cases of more general results. Similarly one can derive a general $D$-dimensional analytical result for the one-loop nondegenerate mass case. 
For three-dimensional space-time we have the dispersion representation

$$
\Pi(x)=\int_{0}^{\infty} \rho\left(m^{2}\right) D_{3}(x, m) d m^{2}=\int_{0}^{\infty} \rho\left(m^{2}\right) \frac{e^{-m x}}{4 \pi x} d m^{2}
$$

with the three-dimensional scalar propagator

$$
D(x, m)=\frac{\sqrt{m x} K_{\frac{1}{2}}(m x)}{(2 \pi)^{3 / 2} x}=\frac{e^{-m x}}{4 \pi x} .
$$

One can invert Eq. (12) and obtains

$$
2 m \rho\left(m^{2}\right)=\frac{1}{2 \pi i} \int_{c-i \infty}^{c+i \infty} 4 \pi x \Pi(x) e^{m x} d x
$$

which is a special case of Eqs. (7) and (8) with

$$
I_{\frac{1}{2}}(z)=\sqrt{\frac{2}{\pi z}} \sinh (z)
$$

where one only needs to retain the $e^{z}$ piece in the hyperbolic sine function. The solution given by Eq. (14) has the appropriate support as a distribution or, equivalently, as an inverse Laplace transform. It vanishes for $m<M=\sum_{i=1}^{n} m_{i}$ since the contour of integration can be closed to the right where there are no singularities of the integrand. Recall that for large $x$ with $\operatorname{Re}(x)>0$ the asymptotic behaviour of the polarization function $\Pi(x)$ is governed by the sum of the masses of the propagators and reads

$$
\Pi(x) \sim \exp (-x M)
$$

For $m>M$ one can close the contour to the left and then the only singularities of $\Pi(x)$ are the poles at the origin of $\Pi(x)$ since it is a product of the propagators of the form of Eqs. (11) and (13). The integration in Eq. (14) then reduces to finding the residues. Indeed,

$$
\Pi(x)=\prod_{i=1}^{n} D_{3}\left(x, m_{i}\right)=\frac{e^{-M x}}{(4 \pi x)^{n}}
$$

and Eq. (14) gives

$$
2 m \rho\left(m^{2}\right)=\frac{1}{2 \pi i} \int_{c-i \infty}^{c+i \infty} 4 \pi x \Pi(x) e^{m x} d x=\frac{1}{2 \pi i(4 \pi)^{n-1}} \int_{c-i \infty}^{c+i \infty} \frac{e^{(m-M) x}}{x^{n-1}} d x .
$$


At $m>M$ one closes the contour of integration to the left and computes the residue at the origin to obtain $(n>1)$

$$
2 m \rho\left(m^{2}\right)=\frac{(m-M)^{n-2}}{(4 \pi)^{n-1}(n-2) !} \theta(m-M) .
$$

This also explains the simplicity of the structure of the spectral density in odd numbers of dimensions of space-time when traditional means are used [7]. In five-dimensional spacetime Eq. (14) is applicable almost without any change because the propagator now reads

$$
D_{5}(x, m)=\frac{(m x)^{\frac{3}{2}} K_{\frac{3}{2}}(m x)}{(2 \pi)^{5 / 2} x^{3}}=\frac{e^{-m x}}{8 \pi^{2} x^{3}}(1+m x) .
$$

Compared to the three-dimensional case the only additional complication is that the degree of the order of the pole at the origin is changed and that one now has a linear combination of terms instead of the simple monomial in three dimensions.

In even number of dimensions one is dealing with a genuine $K$-transform. We discuss in some detail the important case of four-dimensional space-time. For $D=4(\lambda=1)$, Eqs. (22) and (5) give

$$
\Pi(x)=\int \rho\left(m^{2}\right) D_{4}(x, m) d m^{2}=\int \rho\left(m^{2}\right) \frac{m x K_{1}(m x)}{4 \pi^{2} x^{2}} d m^{2},
$$

and Eq. (8) is written as

$$
2 m \rho\left(m^{2}\right)=\frac{1}{\pi i} \int_{c-i \infty}^{c+i \infty} 4 \pi^{2} x^{2} \Pi(x) I_{1}(m x) d x .
$$

All remarks about the behaviour at large $x$ apply here as well. However, the structure of singularities is more complicated than in the odd-dimensional case. In addition to the poles at the origin there is a cut along the negative semi-axis that renders the computation of the spectral density more involved. The cut arises from the presence of the functions $K_{1}\left(m_{i} x\right)$ in the polarization function $\Pi(x)$. Also the asymptotic behaviour of the function $I_{1}(z)$ is more complicated than that of $I_{1 / 2}(z)$. In particular the extraction of the exponentially falling component on the negative real axis is rather tricky. Incidentally, the 
fall-off behaviour of the function $I_{1}(z)$ on the negative real axis can be taken as an illustration of Stokes' phenomenon of asymptotic expansions (see e.g. [9]). While the analytic structure of the representation is quite transparent and the integration can be performed along a contour in the complex plane, there are some subtleties when one wants to obtain a convenient form for numerical treatment [7].

After closing the contour to the left (for $m>M$ ) using the appropriate part of the function $I_{1}(z)$ we obtain

$$
\begin{aligned}
i \pi & \int_{c-i \infty}^{c+i \infty} x^{2} \Pi(x) I_{1}(m x) d x \\
= & -\int_{\epsilon}^{\infty} K_{1}(m r) r^{2}\left(\Pi\left(e^{i \pi} r\right)+\Pi\left(e^{-i \pi} r\right)\right) d r+2 \int_{\epsilon}^{\infty} K_{1}(m r) r^{2} \Pi(r) d r \\
& \quad+\int_{C_{-}}\left(i \pi I_{1}(m z)+K_{1}(m z)\right) \Pi(z) z^{2} d z+\int_{C_{+}}\left(i \pi I_{1}(m z)-K_{1}(m z)\right) \Pi(z) z^{2} d z
\end{aligned}
$$

where the contours $C_{+}$and $C_{-}$are semi-circles of radius $\epsilon$ around the origin in the upper and lower complex semi-plane, respectively. For practical evaluations of $\Pi\left(e^{ \pm i \pi} r\right)$ the following rule for the analytic continuation of McDonald functions is used:

$$
K_{1}\left(e^{ \pm i \pi} m r\right)=-K_{1}(m r) \mp i \pi I_{1}(m r), \quad m r>0 .
$$

Let us add a few remarks on the final representation Eq. (23) which is in a suitable form for numerical integration. We have introduced an auxiliary regularization in terms of a circle of finite radius $\epsilon$ which runs around the origin with its pole-type singularities. The spectral density is independent of $\epsilon$, and the parameter $\epsilon$ completely cancels in the full expression for the spectral density as given by Eq. (23). This must be so since the spectral density is finite for the water melon class of diagrams. Eq. (23) contains no oscillatory integrands (cf. Eq. (99)), and the integration can be safely done numerically. Thus Eq. (23) is a useful alternative representation for the spectral density. In practice the integration over the semi-circles is done by expanding the integrand in $z$ for small $z$ and keeping only terms singular in $\epsilon$. The expansion requires only a finite number of terms and is a purely algebraic operation. Then the singularity in $\epsilon$ exactly cancels against those of the 
remaining integrals. This cancellation can also be done analytically leaving well defined and smooth integrands for further numerical treatment.

Next we turn to the threshold behaviour of the spectral density. Using the results of the above analysis one finds

$$
2 m \rho\left(m^{2}\right) \sim \int^{\infty} K_{1}(m r) \prod_{i} Z_{1}\left(m_{i} r\right) d r
$$

where $Z_{1}\left(m_{i} r\right)$ is either $I_{1}\left(m_{i} r\right)$ or $K_{1}\left(m_{i} r\right)$. The convergence at large $r>0$ is controlled by the factor $\exp (-(m-M) r)$ as in Eq. (18), and the corresponding expansions in the variable $m-M$ in the region $m \sim M$ can be easily constructed.

To summarize, we have described a novel technique to compute the spectral density of any water melon diagram which reduces the calculation to a one-dimensional integral with well known functions in the integrand. Any tensor structure and form factor structure can be easily added without modification of the basic formulae. Different regimes of behavior with respect to mass/momentum expansions can easily be written down in terms of only two dimensionful parameters (one mass and one momentum or two masses) while other parameters are taken as small. The threshold behaviour of the spectral density can be easily investigated based on such a representation. Explicit analytical formulae (with even that last integration being performed) are found in odd space-time dimensions. This allows one to compute any $n$-particle phase space integral for any kind of particles. The only reason that prevents the final integration to be explicitly done in even number of dimensions is that products of Bessel functions are encountered which cannot be integrated in closed form. This forces one to use numerical integrations in the even-dimensional case except for very special cases. Even then the analytic structure of the solution is completely determined which allows for an efficient and reliable numerical treatment of the problem.

All in all we conclude that the problem of computing the class of multi-loop massive water melon diagrams can be considered to be completely solved. 


\section{Acknowledgements}

We would like to thank A. Davydychev for useful discussions. The work is supported in part by the Volkswagen Foundation under contract No. I/73611. A.A. Pivovarov is supported in part by the Russian Fund for Basic Research under contracts No. 96-01-01860 and 97-02-17065.

\section{References}

[1] J. Gasser and M.E. Sainio, Eur. Phys. J. C6 (1999) 297

[2] P. Post and K. Schilcher, Phys. Rev. Lett. 79 (1997) 4088

[3] P. Post and J.B. Tausk, Mod. Phys. Lett. A11 (1996) 2115

[4] F.A. Berends, A.I. Davydychev and N.I. Ussyukina, Phys. Lett. 426 B (1998) 95

[5] K.G. Chetyrkin, J.H. Kühn and A. Kwiatkowski, Phys. Rep. 277 (1996) 189

[6] L. Brücher, J. Franzkowski and D. Kreimer, Report No. MZ-TH-97-35, hep-ph/9710484

[7] S. Groote, J.G. Körner and A.A. Pivovarov, Nucl. Phys. B542 (1999) 515

[8] I.S. Gradstein, I.M. Ryzhik, "Tables of integrals, series, and products", Academic Press, 1994

[9] G.N. Watson, "Theory of Bessel functions", Cambridge 1944.

[10] A.A. Pivovarov, N.N. Tavkhelidze and V.F. Tokarev, Phys. Lett. 132 B (1983) 402

[11] K.G. Chetyrkin and A.A. Pivovarov, Nuovo Cim. 100 A (1988) 899 
[12] A.A. Pivovarov and V.F. Tokarev, Yad. Fiz. 41 (1985) 524

[13] C.S. Meijer, Proc. Amsterdam Akad. Wet. (1940) 599; 702

[14] A. Erdelyi (editor), "Tables of integral transformations", Volume 2, Bateman manuscript project, 1954.

[15] S. Narison and A.A. Pivovarov, Phys. Lett. B327 (1994) 341

[16] E. Mirkes, Report No. TTP-97-39, hep-ph/9711224

[17] D.J. Gross, R.D. Pisarski and L.G. Yaffe, Rev. Mod. Phys. 53 (1981) 43

[18] A.M. Polyakov, Phys. Lett. 72 B (1978) 477

[19] T. Hatsuda, Nucl. Phys. A544 (1992) 27

[20] A.K. Rajantie, Nucl. Phys. B480 (1996) 729

[21] N.N. Bogoliubov and D.V. Shirkov, "Quantum fields", Benjamin, 1983. 